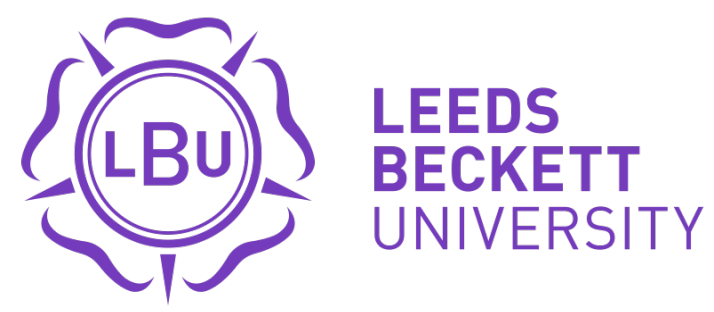

Citation:

Till, $\mathrm{K}$ and Jones, $\mathrm{B}$ and O'Hara, $\mathrm{J}$ and Barlow, $\mathrm{M}$ and Brightmore, $\mathrm{A}$ and Lees, $\mathrm{M}$ and Hind, $\mathrm{K}$ (2016) Three-Compartment Body Composition in Academy and Senior Rugby League Players. International journal of sports physiology and performance, 11 (2). 191 - 196. ISSN 1555-0265 DOI: https://doi.org/10.1123/ijspp.2015-0048

Link to Leeds Beckett Repository record:

https://eprints.leedsbeckett.ac.uk/id/eprint/1482/

Document Version:

Article (Accepted Version)

The aim of the Leeds Beckett Repository is to provide open access to our research, as required by funder policies and permitted by publishers and copyright law.

The Leeds Beckett repository holds a wide range of publications, each of which has been checked for copyright and the relevant embargo period has been applied by the Research Services team.

We operate on a standard take-down policy. If you are the author or publisher of an output and you would like it removed from the repository, please contact us and we will investigate on a case-by-case basis.

Each thesis in the repository has been cleared where necessary by the author for third party copyright. If you would like a thesis to be removed from the repository or believe there is an issue with copyright, please contact us on openaccess@leedsbeckett.ac.uk and we will investigate on a case-by-case basis. 


\section{Title: Three-compartment body composition in academy and senior rugby league players}

3

4
Submission Type: Original Investigation

Kevin Till*, Ben Jones, John O'Hara, Matthew Barlow, Amy Brightmore, Matthew Lees, \& Karen Hind

Research Institute for Sport, Physical Activity and Leisure, Leeds Beckett University, Leeds, West Yorkshire, United Kingdom

*Corresponding Author:

Dr Kevin Till

Room 111, Fairfax Hall

Research Institute for Sport, Physical Activity and Leisure, Headingley Campus, Leeds Beckett University

West Yorkshire, LS6 3QS

Phone: (044-11) 01132-832600 Ext: 25182

Email: k.till@1eedsbeckett.ac.uk

Preferred Running Head: Body composition in rugby league players
Abstract Word Count: 246
Text Only Word Count: 2,760
Number of Tables: 3
Number of Figures: 0 
Abstract

40 Purpose: This study compared the body size and three

41 compartment body composition between academy and senior

42 professional rugby league players using dual energy X-ray

43 absorptiometry (DXA).

44 Methods: Academy (age 18.1 \pm 1.1 years; $n=34$ ) and senior (age

$4526.2 \pm 4.6$ years; $n=63$ ) rugby league players received one total-

46 body DXA scan. Height, body mass and body fat percentage

47 alongside total and regional fat mass, lean mass and bone

48 mineral content (BMC) were compared. Independent t-tests

49 with Cohen's $d$ effect sizes and multivariate analysis of

50 covariance (MANCOVA), controlling for height and body

51 mass, with partial eta squared $\left(\eta^{2}\right)$ effect sizes, were used to

52 compare total and regional body composition.

53 Results: Senior players were taller $(183.2 \pm 5.8$ vs. $179.2 \pm 5.7$

$54 \mathrm{~cm} ; \mathrm{p}=0.001 ; d=0.70)$ and heavier $(96.5 \pm 9.3$ vs. $86.5 \pm 9.0 \mathrm{~kg}$;

$55 \mathrm{p}<0.001 ; d=1.09)$ with lower body fat percentage (16.3 $\pm 3.7 \mathrm{vs.}$

$56 \quad 18.0 \pm 3.7 \% ; \mathrm{p}=0.032 ; d=0.46)$ than academy players.

57 MANCOVA identified significant overall main effects for total

58 and regional body composition between academy and senior

59 players. Senior players had lower total fat mass $(\mathrm{p}<0.001$,

$\left.60 \quad \eta^{2}=0.15\right)$, greater total lean mass $\left(\mathrm{p}<0.001, \eta^{2}=0.14\right)$ and greater

61 total BMC ( $\left.\mathrm{p}=0.001, \eta^{2}=0.12\right)$ than academy players. For

62 regional sites, academy players had significantly greater fat

63 mass at the legs $\left(\mathrm{p}<0.001 ; \eta^{2}=0.29\right)$ than senior players.

64 Conclusions: The lower age, height, body mass and BMC of

65 academy players suggest that these players are still developing

66 musculoskeletal characteristics. Gradual increases in lean mass

67 and BMC whilst controlling fat mass is an important

68 consideration for practitioners working with academy rugby

69 league players, especially within the lower body.

70

71 Key Words: anthropometry, dual energy x-ray absorptiometry

72 (DXA), fat mass, lean mass, bone mineral content 
Introduction

Rugby league is an international collision sport involving frequent periods of high intensity activity separated by lower intensity activity. ${ }^{1}$ Within rugby league, body composition is an important consideration for practitioners due to the requirements of players to have highly developed physiological capacities (e.g., speed, aerobic fitness) ${ }^{2}$ alongside health implications (e.g., reducing injury). Past research has reported lower skinfolds and greater lean mass between elite and semi-elite players, alongside lower skinfolds being associated with greater playing minutes ${ }^{3}$ and physiological capabilities. ${ }^{4}$ Within the United Kingdom (UK), talented rugby league players are recruited to a professional club's academy programme between the ages of 16-19 years. One purpose of an academy programme is to develop the physical qualities of academy rugby league players to meet the increasing training and game demands at higher levels. ${ }^{5,6}$ Therefore, understanding and evaluating the differences in anthropometric and body composition of academy and senior players is of value.

To date, the majority of research examining the body size and body composition (using skinfold assessments) profiles of rugby league players have evaluated the effect of playing level $\mathrm{l}^{3,7}$ and playing position ${ }^{4,8}$ within junior and senior populations. An increase in height and body mass, and a decrease in the sum of skinfolds, is observed at higher playing levels. Reflecting the demands of the game, forwards tend to be taller and heavier with greater skinfold thickness within both junior $^{4,8,9}$ and senior ${ }^{10}$ playing groups. Previous research has emphasized the importance of larger physical attributes in forward positions due to their game demands predominantly requiring a greater number of physical collisions (e.g., tackles, ball carries). ${ }^{4,10}$

Although research has reported differences in physical characteristics between junior and senior levels, no study has directly compared body size and three compartment body composition between academy and senior professional rugby league players as conducted in Australian Rules Football (AFL). ${ }^{11}$ Given that junior players are still experiencing growth and maturation processes, this analysis is important for nurturing long-term health and performance development within junior rugby league players.

Recent studies in rugby league $\mathrm{e}^{12,13}$ and rugby union ${ }^{14,15}$ have utilized dual energy x-ray absorptiometry (DXA) to analyse three-compartment body composition. Whilst the skinfold technique is useful for routine monitoring of body fat in athletes, DXA is a convenient and useful diagnostic tool for acquiring more comprehensive data on bone and body composition. ${ }^{16}$ DXA provides both total and regional values of fat mass, lean mass and bone mineral content (BMC) which allows more accurate and reliable evaluations of body 
125 composition in athletes. ${ }^{17}$ The aim of this study was to

126 characterize and compare the body size and three-compartment

127 body composition of UK academy (Under 19s) and senior

128 professional rugby league players using DXA whilst also

129 considering playing position.

\section{Subjects}

\section{Materials \&Methods}

Sixty-three senior professional players from two

134 European Super League clubs (backs: $n=27$, age 26.0 \pm 4.3

135 years; forwards: $n=36$, age $26.3 \pm 4.9$ years), and 32 academy

136 players from one European Super League club (backs: $n=15$,

137 age $18.1 \pm 1.1$ years; forwards: $n=19$, age $18.2 \pm 1.1$ years),

138 participated in the study. All protocols received institutional

139 ethics approval and players provided written consent.

\section{Procedures}

A cross-sectional research design was used whereby participants were tested during the last phase of the pre-season period (January - February) in a euhydrated state (urine

144 osmolality $\left.<700 \mathrm{mOsmol} \cdot \mathrm{kg}^{-1}\right) .{ }^{18}$ All scans were scheduled on

145 a rest day so activity levels did not affect the scans.

146 Participants wore minimal clothing, with shoes and jewellery

147 removed. Height was measured using a stadiometer (SECA

148 Alpha, Birmingham, UK) to the nearest $0.1 \mathrm{~cm}$ and body mass

149 was measured using calibrated electronic scales (SECA Alpha

150770 , Birmingham, UK) to the nearest $0.1 \mathrm{~kg}$. Each participant

151 received one total body DXA scan on a fan-beam GE Lunar

152 iDXA (Lunar iDXA, GE Medical Systems, UK) using

153 standard or thick mode depending on body size. Participants

154 lay in the supine position on the scanning table with the body

155 aligned with the central horizontal axis. Arms were positioned

156 parallel to the body, with legs fully extended and feet secured

157 with a canvas and Velcro support to avoid foot movement

158 during the scan acquisition.

159 One certified densitometrist led and analyzed all scans

160 following the manufacturer's guidelines for patient

161 positioning. The regions of interest (ROI) were manually

162 placed to enable the appropriate cuts according to the

163 manufacturer's instructions. Defined regions were for the

164 arms, legs and trunk. The appendicular ROI for the arms and

165 legs were defined by cut lines positioned proximally at the

166 coracoid process and superior iliac crest and lower ramus

167 respectively. The trunk region included the pelvis, abdomen

168 and chest. Scan analysis was performed using the Lunar

169 Encore software (Version 15.0). The machine's calibration

170 was checked and passed on a daily basis using the GE Lunar

171 calibration hydroxyapatite and eproxy resin phantom. There

172 was no significant drift in calibration for the study period.

173 Local precision values for our Centre (in healthy adult

174 subjects, aged 34.6 years) are $0.8 \%$ for total fat mass, $0.5 \%$ 
175 for total lean mass, and $0.6 \%$ for total BMC. ${ }^{19}$ Precision of

176 estimation of values for regional fat mass, lean mass and

177 BMC have been previously reported. ${ }^{20}$

\section{Statistical analysis}

180 All statistical analyses were computed using SPSS version 20 (IBM, Armonk, NY, USA). Before analysis, normality and equality of variance of the variables were assessed using a Kolmorgorov-Smirnov test. Independent T-

184 Tests compared body size and body composition parameters

185 between the academy and senior players and between players 186 grouped by playing position (backs $v s$. forwards). Cohen's effect size statistics ${ }^{21}$ were calculated with corresponding $90 \%$ confidence intervals. Effect sizes were interpreted as $<0.2$ (trivial), 0.2-0.6 (small), 0.6-1.2 (moderate), 1.2-2.0 (large) and $>2.0$ (very large). A multivariate analysis of covariance (MANCOVA) compared body composition parameters between academy and senior players, with height and body mass applied as covariates to account for size differences between levels. Following the MANCOVA, univariate analyses were conducted. Effect sizes using partial eta squared $\left(\eta^{2}\right)$ were calculated and interpreted as $0.01=$ small, $0.06=$ medium and $0.14=$ large according to Cohen. ${ }^{22}$

\section{Results}

Table 1 presents the mean and SD for height, body mass and body fat percentage of the academy and senior players, with sub-group comparisons by backs and forwards. Overall, academy players were significantly shorter, lighter and with a higher body fat percentage than senior players. Academy backs were significantly lighter than senior backs but there were no differences for height or body fat percentage. Academy forwards were significantly shorter, lighter with higher body fat percentage than senior forwards. ***Insert Table 1 near here ${ }^{* * *}$

Table 2 presents the total and regional body composition parameters for all players when controlling for height and body mass. MANCOVA analyses between academy and senior players revealed an overall significant effect $\left(\mathrm{F}_{12,82}=5.45, p<0.001, \eta^{2}=0.44\right)$. Univariate analysis identified adjusted differences between academy and senior players for each body composition parameter. Academy players had greater total and regional fat mass, lower lean mass and lower BMC. Specifically, large effect sizes $\left(\eta^{2}=0.29\right)$ were identified for leg fat mass with academy players having greater leg fat mass than senior players.***Insert Table 2 near here $* * *$

Table 3 presents the total and regional body composition parameters for backs and forwards when controlling for height and body mass. MANCOVA analyses between academy and senior forwards revealed an overall 
significant effect $\left(\mathrm{F}_{12,40}=4.61, p<0.001, \eta^{2}=0.58\right)$ but no overall effect was identified for the backs. In forwards, univariate analysis identified significant differences between academy and senior players, favoring the senior players, in all adjusted body composition variables, except arm lean mass and total fat mass, lean mass and BMC alongside arm BMC, leg fat mass, trunk lean mass and trunk BMC where academy players had greater fat mass and lower lean mass and BMC on all occasions.

$$
\text { ***Insert Table } 3 \text { near here }{ }^{* * *}
$$

\section{Discussion}

Knowledge of body size and body composition profiles as they relate to academy and senior professional rugby league players is an important step towards optimizing the long-term development of player performance. This is the first study to evaluate and compare the three-compartment regional body composition profiles of academy and senior rugby league players using DXA. The findings showed that academy players, especially academy forwards, are shorter, lighter with greater body fat percentage than senior players. When height and body mass were controlled, academy players possessed more fat mass, and less lean mass and BMC than senior players. Specifically, academy players have substantially greater fat mass at the legs than senior players.

Height, body mass and body fat percentage differentiated between academy and senior rugby league players. Senior players were taller (ES = moderate) and heavier (ES = moderate-large), likely reflecting that academy players are still experiencing growth, maturation and developmental processes, or a possible talent identification effect at the professional level. These findings are consistent with differences in body mass between junior and senior AFL players. ${ }^{11}$ Research elsewhere has demonstrated greater height and body mass with age between 16 and 20 years $^{23}$ and increases in body mass across a playing season ${ }^{24}$ in academy rugby league players. Given that the average age of the academy players was $18.1 \pm 1.1$ years, it is likely that some players are still developing and may not have attained adult height due to the normal adaptations related to growth in height, which continue to develop into early adulthood. ${ }^{25}$ It is also likely body mass will continue to develop into adulthood, especially with the further inclusion of resistance training (usually from 16 years of age in academy rugby players) and nutrition interventions within an academy programme. ${ }^{23}$ Therefore, differences in height and body mass can be expected between academy and senior players and it is recommended that academy players are regularly monitored for height and body mass into early adulthood. 
For body fat percentage, a small difference was evident

276 between academy and senior players (18.0 \pm 3.7 vs. $16.3 \pm 3.7$

$277 \%$ ). Previous research between players aged 16 and 20 years ${ }^{23}$

278 has shown no difference in sum of four skinfolds by age

279 category, but studies directly assessing body fat percentage are

280 not available. A lower body fat percentage may be advantageous for rugby league performance, as shown through differences reported between Australian elite and semi-elite players, ${ }^{3}$ and relationships between lower sum of skinfolds and playing minutes ${ }^{3}$ and physical characteristics. ${ }^{4}$ Although momentum is an important characteristic for rugby league performance, ${ }^{2,24}$ the ability to accelerate may be compromised by additional fat mass. Therefore, the increasing movement demands of senior rugby league performance ${ }^{6}$ may require professional players to maintain sufficient levels of fat mass to meet the demands of the game. Never-the-less, fat mass may also have beneficial effects for players, ${ }^{26}$ through secretion of bone anabolic hormones from pancreatic beta cells, which may bring faster and more complete recovery from bone micro damage. ${ }^{27}$ In addition, fat mass may provide direct protective effects against fracture, as reported in non-sport populations. ${ }^{28}$ Thus, a certain amount of fat mass may be beneficial for professional players, particularly younger players during peak bone mass accrual, but to date, the exact requirements remain unknown.

Findings between positional groups are consistent with previous research in junior ${ }^{14}$ and senior ${ }^{1}$ players, with forwards reported to be taller, heavier with a greater body fat percentage than backs. For height, only small differences were identified between academy and senior professional backs while moderate differences were identified for forwards. This suggests height may be a more important characteristic within forward positions and more likely used within identification processes for forwards. For body mass, senior professional players were heavier (ES = moderate-large) for both backs and forwards, suggesting that increased body mass is an important consideration for the development of junior players into senior professionals in all positions. For body fat percentage, senior professional forwards were leaner $(17.2 \pm 3.7$ vs.19.8 $\pm 3.1 \%$; $\mathrm{ES}=$ moderate) than academy forwards with only trivial effects observed between academy and senior professional backs. Although forwards usually have a greater body fat percentage than backs due to the contact demands of the position, this finding suggests that it may be advisable for body fat to be monitored in academy forwards for optimal player development in terms of progressing to professional levels. Longitudinal research would be valuable to determine the extent and time course of body composition shifts, and in relation to injury incidence, particularly in forwards progressing from academy to senior professional level. 
The lower fat mass, and greater lean mass and BMC of senior professional players, when height and body mass were controlled, is suggestive of attainment of musculoskeletal maturity and increased training and match demands. ${ }^{6}$ The larger distances covered at high intensity running speeds, increased repeated high intensity efforts together with the contact and collision nature of the sport, would emphasise increased lean mass and appropriate level of fat. ${ }^{1,5}$ In terms of growth and maturation, although height velocity plateaus in late adolescence, lean mass and BMC continues to increase into the early $20 \mathrm{~s} .{ }^{29}$ As such, academy players are likely to be still undergoing natural growth processes at completion of a UK academy programme (i.e., 19 years of age) and into the early years of competing at senior professional levels. This should be considered by coaches and player development staff for player recruitment and long-term player development.

This is the first study to evaluate both total and regional three-compartment body composition profiles in rugby league players, with previous research only available in rugby union and Sevens players. ${ }^{15}$ Quantifying regional distributions may inform physical developmental priorities for junior and senior players. Comparisons found differences between academy and senior professional players between regions for fat mass, lean mass and BMC that correspond with the overall findings that senior professional players have greater lean mass and BMC but reduced fat mass in each region. Interestingly a large difference was observed in leg fat mass between academy and senior professional players. This suggests that the development processes at this age are characterized by greater fat mass in the lower body during growth and maturation, or that advanced training and playing interventions at senior level may reduce fat mass within the lower body. Without a control group or longitudinal investigation it is difficult to confirm this or ascertain the mechanisms involved. However, due to the importance of the legs for optimizing rugby specific actions such as ball carrying, tackling and strength and power related activity $^{2}$ this may be an important consideration for monitoring and training purposes.

Although this study has developed upon previous body composition research within rugby league, limitations do exist. Participants were not fasted on testing, which increases the error of measurement of body mass and lean mass within DXA scans, ${ }^{17}$ possibly questioning the differences between academy and professional players. The cross-sectional nature of the study means that body size and body composition can only be determined acutely. Evaluating longitudinal changes in players' body composition from academy to senior professional level would be valuable to further inform on the role of fat mass, lean mass and BMC for the optimal development in rugby league. Finally, the inclusion of a control group would have 
enabled greater insights into natural, age-related developments in body size and composition.

\section{Practical Applications}

These findings demonstrate that body size and body composition profiles differ between academy and senior professional rugby league players and are therefore an important consideration for junior player development. Practitioners should be aware that academy players are developing musculoskeletal characteristics and may still be experiencing such processes when participating in a rugby academy at 19 years of age. Greater differences also seem apparent between academy and senior players within the forwards position. Such processes may therefore affect player recruitment and development strategies. Practitioners should consider the gradual development of lean mass and BMC whilst controlling fat mass in academy players on progress into senior professional competition, especially within the forward position. It is recommended that practitioners monitor body size and body composition of players regularly into the early twenties employing standardized protocols when using DXA. ${ }^{30}$

\section{Conclusions}

This is the first study to compare the body size and body composition differences between academy and senior professional rugby league players using DXA. Differences were evident favoring the senior players suggesting academy players may still be developing physically into early adulthood. Given that greater lean mass and lower body fat are related to physical ability and game performance in rugby league, the development of these characteristics should be considered, but alongside the impact upon health status (i.e. bone mass, injury and injury prevention, illness). Further research evaluating longitudinal changes in body composition profiles is required to provide a greater understanding of this development process and the individual effects of lean and fat mass on performance, career longevity and health in this population. 


\section{References}

1. Johnston RD, Gabbett TJ, Jenkins DJ. Applied sport science of rugby league. Sports Med. 2014; 44:10871100

2. Baker DG, Newton RU. Comparison of lower body strength, power, acceleration, speed, agility, and sprint momentum to describe and compare playing rank among professional rugby league players. J Strength Cond Res. 2008; 22:153-158.11.

3. Gabbett TJ, Jenkins DG, Abernethy B. Relationships between physiological, anthropometric, and skill qualities and playing performance in professional rugby league players. J Sports Sci. 2011;29:1655-1664.

4. Till K, Cobley S, O'Hara J, Cooke C, Chapman C. Anthropometric, physiological and selection characteristics in high performance UK junior rugby League players. Talent Dev Excellence. 2010;2:193207.

5. Black GM, Gabbett TJ. Repeated High-Intensity Effort Activity in Elite and Semi-Elite Rugby League MatchPlay. Int J Sports Physiol Perform. 2014 Jul 22. [Epub ahead of print]

6. Gabbett TJ. Influence of playing standard on the physical demands of professional rugby league. $J$ Sports Sci. 2013;31:1125-1138.

7. Till K, Cobley S, O'Hara J, Brightmore A, Chapman C, Cooke C. Using, anthropometric and performance characteristics to predict selection in junior UK rugby league players. J Sci Med Sport. 2011;14:264-269.

8. Cheng HL, O'Connor H, Kay S, Cook R, Parker H, Orr R. Anthropometric characteristics of Australian junior representative rugby league players. J Sci Med Sport. 2014; 17:546-551

9. Till K, Cobley S, O’Hara J, Chapman C, Cooke C. A longitudinal evaluation of anthropometric and fitness characteristics in junior rugby league players. J Sci Med Sport. 2013;16:438-443.

10. Morgan PJ, Callister R. Effects of a preseason intervention on anthropometric characteristics of semi professional rugby league players. J Strength Cond Res. 2011;25:432-40.

11. Veale JP, Pearce AJ, Buttifant D, Carlson JS Anthropometric profiling of elite junior and senior Australian football players. Int J Sports Physiol Perform. 2010;5:509-20.

12. Georgeson EC, Weeks BK, McLellan C, Beck BR. Seasonal change in bone, muscle and fat in professional rugby league players and its relationship to injury: a cohort study. BMJ Open 2012;2: e001400. doi:10.1136/bmjopen-2012-001400 
13. Harley JA, Hind K, O'Hara JP. Three-compartment body composition changes in elite rugby league players during a super league season, measured by dual-energy X-ray absorptiometry. J Strength Cond Res. 2011;25:1024-9.

14. Delahunt E, Byrne RB, Doolin RK, McInerney RG, Ruddock CT, Green BS.Anthropometric profile and body composition of Irish adolescent rugby union players aged 16-18. J Strength Cond Res. 2013;27:3252-3258.

15. Higham DG, Pyne DB, Anson JM, Dziedzic CE, Slater GJ. Distribution of fat, non-osseous lean and bone mineral mass in international rugby union and rugby sevens players. Int J Sports Med. 2014;35:575-582.

16. Toombs RJ, Ducher G, Shepherd JA, De Souza MJ. The impact of recent technological advances on the trueness and precision of DXA to assess body composition. Obesity. 2012;20:30-39.

17. Nana A, Slater GJ, Hopkins WG, Burke LM. Effects of daily activities on dual-energy X-ray absorptiometry measurements of body composition in active people. Med Sci Sports Exerc 2012;44:180-189

18. Shirreffs SM, Maughan RJ. Urine osmolality and conductivity as indices of hydration status in athletes in the heat. Med Sci Sports Exerc. 1998;30:1598-602

19. Hind K, Oldroyd B, Truscott J. In-vivo short term precision of the GE Lunar iDXA for the measurement of three compartment total body composition in adults. Eur J Clin Nutr. 2011; 65:140-142

20. Hind K, Oldroyd B. In-vivo precision of the GE Lunar iDXA densitometer for the measurement of appendicular and trunk lean and fat mass. Eur J Clin Nutr. 2013; 67: 1331-1333

21. Batterham AM, Hopkins WG. Making inferences about magnitudes. Int J Sports Physiol Perform. 2006; 1:5057

22. Cohen, J. Statistical Power Analysis for the Behavioral Sciences (2nd ed.). New Jersey, NJ: Lawrence Erlbaum, 1988.

23. Till K, Tester E, Jones B, Emmonds S, Fahey J, Cooke C. Anthropometric and Physical Characteristics of English Academy Rugby League Players. J Strength Cond Res. 2014; 28:319-327

24. Till K, Jones B, Emmonds S, Tester E, Fahey J, Cooke C. Seasonal changes in anthropometric and physical characteristics within English academy rugby league players. J Strength Cond Res. 2014; 28: 2689-2696.

25. Malina RM, Bouchard C, Bar-Or O. Growth, Maturation, and Physical Activity (2nd ed.). Champaign, IL: Human Kinetics, 2004. 
26. Hind K, Gannon L, Brightmore A, Beck B. Insights into

relationships between body mass and bone: findings in elite rugby players. J Clin Densitom. In press

27. Reid IM. Relationships between fat and bone.

28. Reid IM. Fat and Bone. Arch Biochem BioPhys 2010;503:20-27

29. Molgaard C, Thomsen BL, Prentice A, Cole T, Michaelsen KF. Whole body bone mineral content in healthy children and adolescents. Archives of Disease in Childhood 1997;76:9-15.

30. Nana A, Slater GJ, Hopkins WG, Halson SL, Martin DT, West NP, et al. Importance of Standardized DXA Protocol for Assessing Physique Changes in Athletes. Int J Sport Nutr Exerc Metab 2014; Epub. 
Table 1. Differences in height, body mass and body fat percentage between Academy $(n=32)$ and Professional (n=63) rugby league players grouped by playing position (mean + SD)

\begin{tabular}{lcccc}
\hline & Academy & Professional & $\boldsymbol{P}$ & Cohen's d (90\% CIs) \\
\hline All Players & & & & \\
Height (cm) & $179.2 \pm 5.7$ & $183.2 \pm 5.8$ & 0.001 & $0.70[0.32-1.05]$ \\
Body Mass (kg) & $86.5 \pm 9.0$ & $96.5 \pm 9.3$ & $<0.001$ & $1.09[0.70-1.46]$ \\
Body Fat Percentage & $18.0 \pm 3.7$ & $16.3 \pm 3.7$ & 0.032 & $0.46[0.09-0.82]$ \\
Backs & & & & \\
Height (cm) & $178.5 \pm 6.4$ & $181.7 \pm 5.9$ & 0.11 & $0.52[0.16-0.89]$ \\
Body Mass (kg) & $82.1 \pm 7.5$ & $91.3 \pm 8.6$ & 0.001 & $1.14[0.73-1.43]$ \\
Body Fat Percentage & $15.8 \pm 3.1$ & $15.2 \pm 3.4$ & 0.60 & $0.18[-0.18-0.54]$ \\
Forwards & & & & \\
Height (cm) & $179.7 \pm 5.2$ & $184.4 \pm 5.6$ & 0.004 & $0.87[0.48-1.22]$ \\
Body Mass (kg) & $89.9 \pm 8.8$ & $100.4 \pm 7.8$ & $<0.001$ & $1.26[0.89-1.67]$ \\
Body Fat Percentage & $19.8 \pm 3.1$ & $17.2 \pm 3.7$ & 0.01 & $0.76[0.37-1.10]$ \\
\hline
\end{tabular}


Table 2: Adjusted differences in total and regional body composition between academy and professional rugby league players presented as the mean ( $95 \%$ CIs), with covariates height and body mass.

\begin{tabular}{lccccc} 
& Academy & Professional & Difference & $\boldsymbol{P}$ & $\boldsymbol{\eta}^{2}$ \\
\hline Total & & & & & \\
Fat Mass (kg) & $17.1(1.2)$ & $14.1(0.8)$ & 3.0 & $<0.001$ & 0.15 \\
Lean mass (kg) & $71.8(1.0)$ & $74.6(0.8)$ & -2.8 & $<0.001$ & 0.14 \\
BMC (g) & $4081(101)$ & $4313(71)$ & -232 & 0.001 & 0.12 \\
Regional & & & & & \\
Arms Fat Mass (kg) & $1.78(0.12)$ & $1.54(0.09)$ & 0.24 & 0.003 & 0.09 \\
Arms Lean mass (kg) & $9.6(0.3)$ & $10.0(0.2)$ & -0.4 & 0.017 & 0.06 \\
Arms BMC (g) & $575(19)$ & $631(13)$ & -56 & $<0.001$ & 0.19 \\
Legs Fat Mass (kg) & $6.2(0.4)$ & $4.6(0.2)$ & 1.6 & $<0.001$ & 0.29 \\
Legs Lean mass (kg) & $24.6(0.5)$ & $25.3(0.4)$ & -0.7 & 0.033 & 0.05 \\
Legs BMC (g) & $1537(38)$ & $1613(27)$ & -76 & 0.004 & 0.09 \\
Trunk Fat Mass (kg) & $8.1(0.7)$ & $7.0(0.5)$ & 1.1 & 0.015 & 0.06 \\
Trunk Lean mass (kg) & $34.2(0.7)$ & $35.8(0.5)$ & -1.6 & 0.001 & 0.12 \\
Trunk BMC (g) & $1380(39)$ & $1466(28)$ & -86 & 0.001 & 0.11 \\
\hline Note: $\eta^{2}-0.01=5 m 11$
\end{tabular}

Note: $\eta^{2}-0.01=$ small, $0.06=$ medium and $0.14=$ large; $\mathrm{BMC}=$ Bone Mineral Content 
Table 3: Adjusted differences in total and regional body composition between Academy and professional rugby league players by playing position presented as the mean (95\% CIs), with covariates height and body mass.

\begin{tabular}{|c|c|c|c|c|c|c|c|c|c|c|}
\hline & \multicolumn{5}{|c|}{ Backs } & \multirow[b]{2}{*}{ Academy } & \multirow[b]{2}{*}{ Professional } & \multicolumn{3}{|c|}{ Forwards } \\
\hline & Academy & Professional & Diff & $\boldsymbol{P}$ & $\eta^{2}$ & & & Diff & $\boldsymbol{P}$ & $\eta^{2}$ \\
\hline \multicolumn{11}{|l|}{ Total } \\
\hline Fat Mass (kg) & $13.7(1.6)$ & $12.6(1.1)$ & 1.1 & 0.307 & 0.03 & $19.3(1.6)$ & $15.4(1.1)$ & 3.9 & $<0.001$ & 0.22 \\
\hline Lean mass (kg) & $70.3(1.6)$ & $71.3(1.1)$ & -1.0 & 0.346 & 0.02 & $73.3(1.5)$ & $76.9(1.1)$ & -3.6 & 0.001 & 0.20 \\
\hline $\mathrm{BMC}(\mathrm{g})$ & 4009 (139) & 4135 (99) & -126 & 0.172 & 0.05 & 4157 (153) & 4435 (105) & -278 & 0.007 & 0.14 \\
\hline \multicolumn{11}{|l|}{ Regional } \\
\hline Arms Fat Mass (kg) & $1.45(0.16)$ & $1.41(0.12)$ & 0.04 & 0.677 & 0.01 & $1.99(0.18)$ & $1.66(0.12)$ & 0.33 & 0.008 & 0.13 \\
\hline Arms Lean mass (kg) & $9.3(0.4)$ & $9.5(0.3)$ & -0.2 & 0.290 & 0.03 & $9.9(0.4)$ & $10.3(0.2)$ & -0.4 & 0.086 & 0.06 \\
\hline Arms BMC (g) & $562(31)$ & $602(22)$ & -42 & 0.046 & 0.10 & $588(26)$ & $652(18)$ & -64 & $<0.001$ & 0.23 \\
\hline Legs Fat Mass (kg) & $4.9(0.6)$ & $4.2(0.4)$ & 0.7 & 0.072 & 0.08 & $7.1(0.6)$ & $5.1(0.4)$ & 2.0 & $<0.001$ & 0.41 \\
\hline Legs Lean mass (kg) & $24.1(0.9)$ & $24.0(0.6)$ & 0.1 & 0.853 & 0.00 & $25.1(0.6)$ & $26.2(0.4)$ & -1.2 & 0.01 & 0.12 \\
\hline Legs BMC (g) & $1518(58)$ & $1566(41)$ & -48 & 0.206 & 0.04 & $1569(54)$ & $1639(37)$ & -70 & 0.054 & 0.07 \\
\hline Trunk Fat Mass (kg) & $6.4(1.0)$ & $6.1(0.7)$ & 0.3 & 0.620 & 0.01 & $9.3(1.1)$ & $7.8(0.8)$ & 1.5 & 0.032 & 0.09 \\
\hline Trunk Lean mass (kg) & $33.6(1.0)$ & $34.5(0.8)$ & -0.9 & 0.232 & 0.04 & $34.7(1.0)$ & $36.8(0.8)$ & -2.1 & 0.005 & 0.15 \\
\hline Trunk BMC (g) & $1362(51)$ & $1391(38)$ & -29 & 0.398 & 0.02 & $1400(59)$ & $1520(40)$ & -120 & 0.003 & 0.16 \\
\hline
\end{tabular}

Note: $\eta^{2}-0.01=$ small, $0.06=$ medium and $0.14=$ large $; \mathrm{BMC}=$ Bone Mineral Content 
